\title{
Recolección y análisis de datos en el ámbito de la eficiencia energética eléctrica
}

\author{
Data collection and analysis for electric energy efficiency
}

Hernán Santiso ${ }^{1}$, Martín Salamero ${ }^{2}$, Cristian Novarino ${ }^{3}$

Resumen.- El crecimiento en el uso de nuevas tecnologías en los sistemas de medición de consumo eléctrico ha hecho posible el surgimiento de grandes oportunidades de mejora en la gestión energética.

En este proyecto se ha realizado un estudio de aplicación de estas tecnologías con el objeto de colaborar con la diversificación de este tipo de dispositivos a través de soluciones técnicas económicas que puedan ser incorporadas en las viviendas de manera sencilla. De esta forma las personas podrán conocer sus patrones de consumo y detectar oportunidades de ahorro.

Como resultado del trabajo se presenta un prototipo técnico abierto y de bajo costo, que incluye los componentes necesarios para recolectar datos de consumo residencial y volcarlos a un sistema informático de nube, donde los usuarios pueden acceder a información detallada del consumo de los dispositivos que utiliza a diario.

Palabras clave: Eficiencia Energética, control de consumo eléctrico, Medición energía bajo costo.

Summary.- The growth of the use of electricity consumption measurement systems new technologies has made possible the emergence of great opportunities to improve energy management.

The project aims to study the application of these technologies, in order to collaborate with the diversification of this type of devices through economic technical solutions that can be easily included in the houses. In this way, people will be able to know their consumption patterns and detect savings opportunities.

As a result of this work, an open and low-cost technical prototype is presented, including the necessary components to collect home electrical consumption data and upload it to a cloud computing system in order to allow the users access to daily used devices consumption detailed information.

Keywords: Efficience Energy, Electric consumption control, low-cost energy measurement.

1. Introducción.- Hace ya algunos años se ha comenzado a tomar conciencia a nivel mundial acerca del efecto adverso para la ecología que provoca el uso de combustibles fósiles para la generación de energía eléctrica. Sumado a esto el mundo está atravesando por un proceso

\footnotetext{
${ }^{1}$ Facultad de Ing., Universidad Blas Pascal, hsantiso@hotmail.com, ORCID iD: 0000-0001-5500-8892

${ }^{2}$ Facultad de Ing., Universidad Blas Pascal, martin.salamero@gmail.com, ORCID iD: 0000-0001-9174-5937

${ }^{3}$ Facultad de Ing., Universidad Blas Pascal, novacrez@gmail.com, ORCID iD: 0000-0002-4034-3177
} 
sistemático de encarecimiento del costo de las tarifas de servicio que hace que las iniciativas de ahorro y uso eficiente de la energía sean de mucho interés por su campo de aplicación.

Por otro lado, el crecimiento de las tecnologías relacionadas con el Internet de las Cosas, ha facilitado el desarrollo de plataformas de Big data con capacidad de procesamiento de datos extremadamente alta. Sobre dichas plataformas se puede enviar las mediciones recolectados por multiplicidad de diferentes sensores para realizar correlaciones, y establecer modelos de comportamientos que permitan entender y controlar los procesos físicos.

Estos avances nos ofrecen nuevas oportunidades para mejorar la gestión energética a través del procesamiento de mayor cantidad de datos y obtención de información de valor que permita tomar decisiones con mayor nivel de certeza casi en tiempo real, pudiendo detectar anomalías, definir patrones de consumo, anticipar demandas y realizar correcciones y ajustes de manera más oportuna.

A pesar de ello, aún no se vislumbra que estos adelantos estén accesibles al común de la gente, quedando solamente reservada su aplicación en grandes organizaciones que poseen una capacidad de inversión apropiada para implementar estas soluciones o en proyectos especiales en el ámbito privado asociados a la reducción de costos de operación.

En estos últimos años han aparecido un conjunto de dispositivos hogareños tendientes a cubrir esta problemática, pero el costo de los mismos es un tanto elevado y sumado a eso, los datos colectados sólo pueden ser procesados por el proveedor de servicio, dejando de lado del usuario sólo la posibilidad de acceder a los datos de forma limitada.

Es por todo lo expuesto anteriormente, que el objetivo de este trabajo es el de crear un prototipo técnico de recolección y compilación de métricas de consumo de energía eléctrica, que brinde acceso a toda la información recogida a través del uso de tecnologías abiertas, de bajo costo y escalables para el uso masivo.

Un desarrollo de estas características puede ser de gran valor para la comunidad en general ya que posibilitará a los usuarios llevar una contabilidad sobre sus consumos eléctricos, y poder hacer un uso más eficiente de los de la energía reduciendo además los costos del servicio.

2. Desarrollo del trabajo.- El proyecto fue dividido en dos etapas principales. En la primera se realizó una investigación del estado actual de situación en cuanto a la gestión de la eficiencia energética, los modelos de arquitectura de las tecnologías de grandes datos y los sistemas de medición y gestión energética para poder determinar los casos de uso reales donde estas soluciones tienen mayor aplicabilidad.

Una vez hecho esto se procedió a crear un prototipo tecnológico abierto y de bajo costo con los componentes necesarios para que la información de consumo energético pueda ser volcada a un sistema de análisis que soporte gran cantidad de información.

Por último, se realizaron pruebas técnicas sobre el del prototipo desarrollado, con el objeto de verificar su utilidad y correcto funcionamiento, con el fin de facilitar la difusión y adopción de estas soluciones por la comunidad en general.

\subsection{Relevamiento del estado de situación.-}

2.1.1. La Eficiencia Energética y su relación con el análisis de datos.- Según el informe 
"Indicadores de políticas públicas en materia de eficiencia energética en América latina y el Caribe" [1] establece dos definiciones básicas de eficiencia.

La primera se basa en un concepto bastante utilizado en la ingeniería de energía y es recíproco al consumo específico, expresando una productividad o una relación beneficio/costo. Así, una alta eficiencia energética representa la capacidad de obtener una producción de bienes o servicios con bajo consumo energético.

Otra forma de definir "Eficiencia Energética" se asocia a la eficiencia económica, refiriéndose a una reducción en la energía utilizada para un mismo nivel de satisfacción de un requerimiento energético final, incluyendo los cambios tecnológicos, económicos, institucionales y de comportamiento asociados.

Una manera general de definir la eficiencia energética de un equipo o sistema energético es como el cociente entre flujos energéticos útiles y deseables producidos y los flujos consumidos.

$$
\text { Eficiencia energética }=\left(\frac{\text { Efecto energético útil deseado }}{\text { Consumo energético }}\right) \times \text { Equipo o proceso }
$$

Hay sistemas que producen flujos energéticos útiles que no son utilizados, entonces una definición equivalente podría relacionar los flujos energéticos aprovechados y consumidos.

$$
\text { Eficiencia energética }=\left(\frac{\text { Energía aprovechada }}{\text { Energía consumida }}\right) \times \text { Equipo o proceso }
$$

Este es un aspecto interesante en el estudio de la Eficiencia Energética, pues permite esclarecer por qué la relación entre el uso racional de energía y la mitigación de las pérdidas debe ser una meta permanente. Hay dos grandes grupos de pérdidas energéticas: a) pérdidas irreversibles (o inevitables), b) reversibles (o evitables frente a procesos ideales).

Reducir las pérdidas energéticas a niveles aceptables implica necesariamente actuar en todos estos frentes, considerando naturalmente los limitantes económicos, pudiendo incorporar beneficios ambientales y otras externalidades.

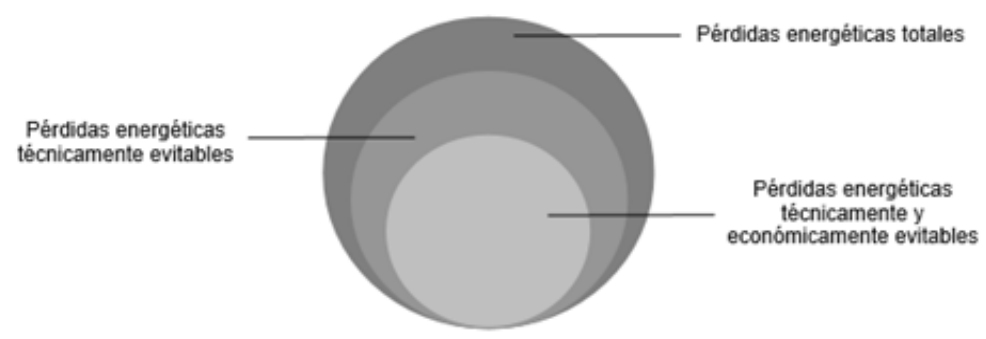

Figura I.- Pérdidas energéticas totales y evitables.

El consumo de un sistema socioeconómico o de un sector en particular, depende de múltiples factores o variables, lo cual hace difícil establecer las relaciones o ecuaciones que vinculan dicho consumo con las variables explicativas. Podemos decir que el uso de la energía depende en 
mayor o menor medida de las siguientes características:

- Ambientales: clima, temperatura, humedad

- Socio-económicas: tamaño de población, condiciones edilicias y uso del espacio, situación económica.

- Energéticas: acceso y abastecimiento o no de determinadas fuentes energéticas, estructura por fuentes del consumo energético, precios de la energía y los artefactos y equipos de utilización de la misma y EE.

- Pautas culturales

Si conocemos con más exactitud estos datos podremos trabajar en las causas para lograr cambios que mejoren la relación de eficiencia. En especial lo que tiene que ver con conductas y cambios en la forma de utilizar la energía como así también controlar mejor desvíos, derroches y uso inapropiado de la energía.

A continuación, se recorren algunos de los diferentes casos de uso aplicables que se han podido encontrar en los distintos trabajos de investigación revisados, poniendo énfasis en los aportes que estas técnicas pueden realizar a la gestión de la eficiencia energética, siempre desde el punto de vista del consumo eléctrico.

2.1.2. Posibles casos de uso aplicables a eficiencia energética.- Con el objeto de determinar qué casos de uso relacionados con el análisis de datos pueden colaborar con la eficiencia energética, se procedió a revisar y resumir publicaciones existentes en la materia, ya que existe una vasta experiencia comprobada en este campo. Determinar patrones de consumo y su relación con distintas variables ha sido el foco de numerosos estudios revisados [2-6] entre los que se destacan como principales factores los siguientes:

- $\quad$ Tipo de usuario (residencial o industrial)

- Consumo anormal (asociado a fraude)

- Ocupación edilicia

- Relación consumo potencia real vs aparente

- Día laborable o no laborable

- Hora del día (Amanecer y ocaso)

- Temperatura ambiente, época del año

- $\quad$ Tipos de dispositivos utilizados

- Patrones incrementales.

El análisis de estos y otros aspectos relacionados al consumo son los que conformarán los puntos de partida para la aplicación de la analítica de datos a través de diferentes acciones como por ejemplo la detección temprana de desvíos, conductas anormales o problemas técnicos, la anticipación de la demanda y la retroalimentación para generar conciencia en el uso racional de la energía. Para ello pueden aplicarse distintas técnicas de análisis de datos, ampliamente difundidas como por ejemplo las técnicas de agrupamiento de datos (clustering), métodos autoregresivos, redes neuronales y bosques aleatorios.

Si bien no se encuentra dentro del alcance de este trabajo realizar este tipo de análisis, se debe garantizar que la información obtenida y capturada pueda ser incluida en los mismos para garantizar su correcto procesamiento.

3. Creación del prototipo.- Una vez comprendidos los casos de uso de análisis de datos aplicables a la mejora de la eficiencia energética fue necesario encontrar una solución técnica que 
permita la recolección y compilación de las métricas de consumo obtenidas, haciendo posible la recolección de dicha información en un repositorio único para su posterior visualización y análisis.

Si bien existen en el mercado múltiples soluciones y dispositivos comerciales que permiten realizar las mediciones de magnitudes eléctricas y recolectarlas para su posterior acceso, no fue posible hallar uno que cumpla con las características necesarias para ser de utilidad en este proyecto y el trabajo a futuro, de acuerdo a las siguientes premisas de diseño:

- Medición de la tensión instantánea

- Medición de la corriente instantánea

- Comunicación mediante Wi-Fi y red celular (GPRS)

- Bajo costo

- Utilización de software y hardware abierto (licencias open source).

- Completamente configurable en lo relativo al envío de datos, intervalos de medición, etc.

- Acceso a los datos en crudo para su posterior análisis.

- Capacidad de almacenar y procesar grandes cantidades de datos (BigData)

El modelo propuesto consta dos partes: en la primera de ellas se presenta la arquitectura necesaria para colectar, procesar y analizar los datos medidos en un sistema informático de nube que soporte el procesamiento de grandes datos y en la segunda se detalla el modelo técnico propuesto para implementar un elemento de medición local que pueda tomar métricas de consumo en una instalación objeto de estudio y enviar los valores obtenidos al mencionado sistema.

En el siguiente gráfico (figura II) se puede observar los distintos componentes y sus interacciones.

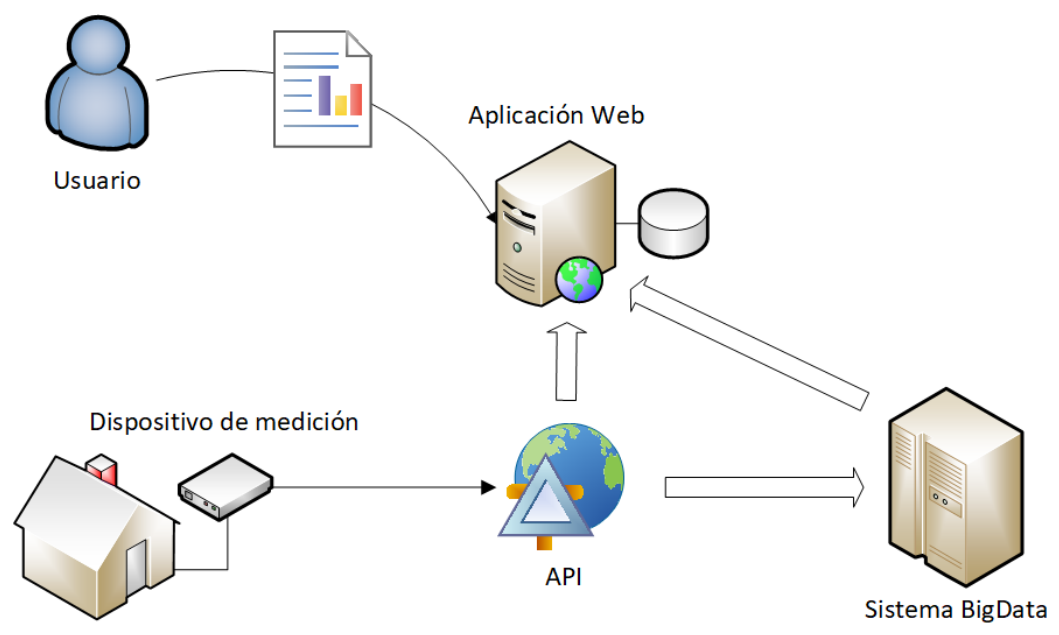

Figura II.- Componentes de un sistema de recolección y análisis de datos de consumo de energía eléctrica.

En esencia la arquitectura del sistema está compuesta por un Programa de Interfaz de Aplicación (API o Application Program Interface), el cual se encarga de recibir los datos del sistema de medición de energía y redireccionar los mismos hacia un sistema de almacenamiento de Big Data.

A su vez se incluye una aplicación web que estará en constante comunicación con su propia base 
de datos, y que se encargará de procesar los datos recibidos e informar al usuario el estado de procesamiento como así también servir de interfaz para acceder a la información recolectada.

3.1. Detalles técnicos de la solución.- A continuación, se describen en detalle los distintos componentes utilizados para conformar la solución técnica de medición y análisis de datos:

\section{$\underline{\text { Servidor Big Data }}$}

Dentro de las opciones se seleccionó InfluxDB [7] en su versión de código abierto ya que es fácil de configurar y es más específico para el tipo de datos a analizar (sistema de software que está optimizado para manejar datos de series de tiempo, matrices de números indexados por tiempo).

\section{API y Aplicación WEB}

Se utilizó el framework Laravel [8] porque es un marco de desarrollo en lenguaje php que brindan una rápida solución para el desarrollo. Como base de datos de la aplicación se utilizó MariaDB [9] ya que tiene una mejor respuesta en velocidad comparado con MySQL [10].

\section{Dispositivo de medición de energía eléctrica}

El componente principal del equipo de medición es el sensor, que es un objeto capaz de variar una propiedad ante magnitudes físicas o químicas, llamadas variables de instrumentación, y transformarlas con un transductor en variables eléctricas. Las variables de instrumentación pueden ser, por ejemplo: intensidad lumínica, temperatura, distancia, aceleración, inclinación, presión, desplazamiento, fuerza, torsión, humedad, movimiento, $\mathrm{pH}$, etc.

El resultado final del diseño incorpora los siguientes elementos principales:

1) PZEM-004T: Es el hardware encargado de la medición de la corriente circulando y la tensión alterna presente en el circuito del ambiente a monitorear. Es una placa con su propio microcontrolador y circuitos asociados, que mediante una bobina toroidal realiza la medición de la corriente consumida, y mediante una resistencia de carga efectúa la medición de voltaje. Toda esta información puede ser recolectada y transmitida por un puerto serie. En la figura III se ilustra el componente PZEM-004T.

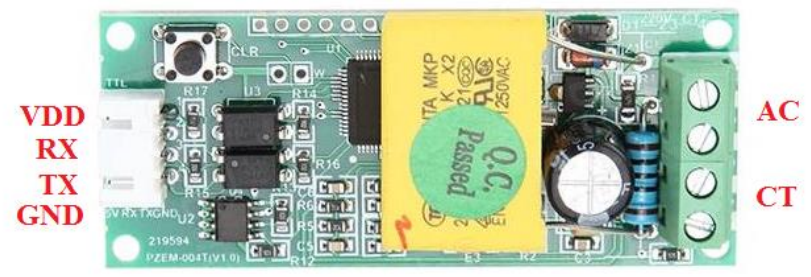

Figura III.- Componente PZEM-004T.

2) ESP8266: Es una placa de bajo costo que incorpora comunicación Wi-Fi completa y un microcontrolador programable y librerías disponibles en el entorno de desarrollo Arduino [11] el cual facilita el trabajo con el mismo. En este proyecto Se utilizó como unidad central, es decir que se programó para interpretar la información proveniente del sensor PZEM-004T mediante puerto serie, gestionando además las comunicaciones Wi-Fi y GPRS. En la figura IV se ilustra el componente ESP8266. 


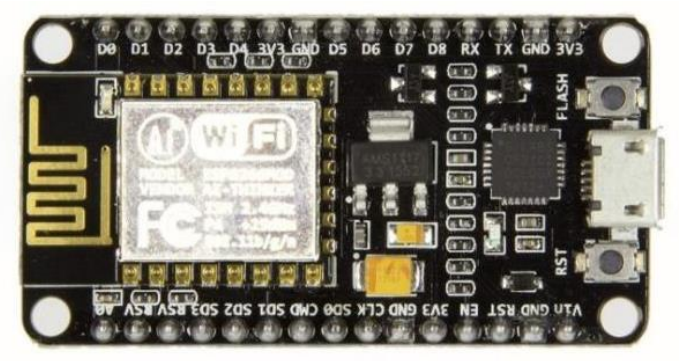

Figura IV.- Componente ESP8266.

3) SIM 900: Es un módulo autónomo de GPRS que se conecta a la red de telefonía móvil de cualquier operador con la sola utilización de un chip GSM, en este proyecto este dispositivo es controlado por el ESP8266 para efectuar el envío de la información de corriente y tensión recabada al servidor encargado de interpretar esos datos. En la figura V se ilustra el componente SIM 900.

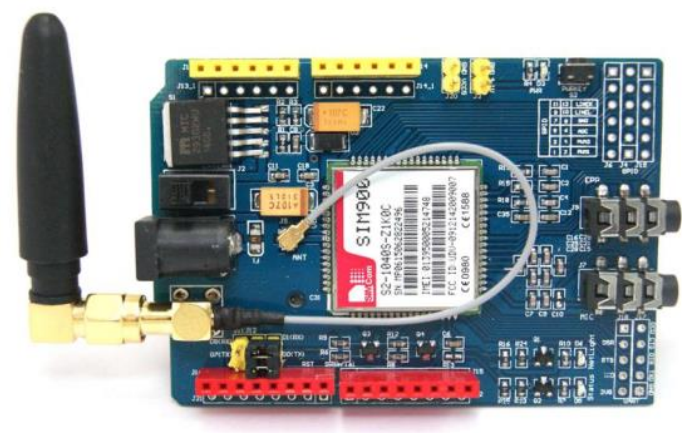

Figura V.- Componente SIM 900.

A continuación, en la figura VI se muestra la estructura operativa del sistema de medición de energía eléctrica:

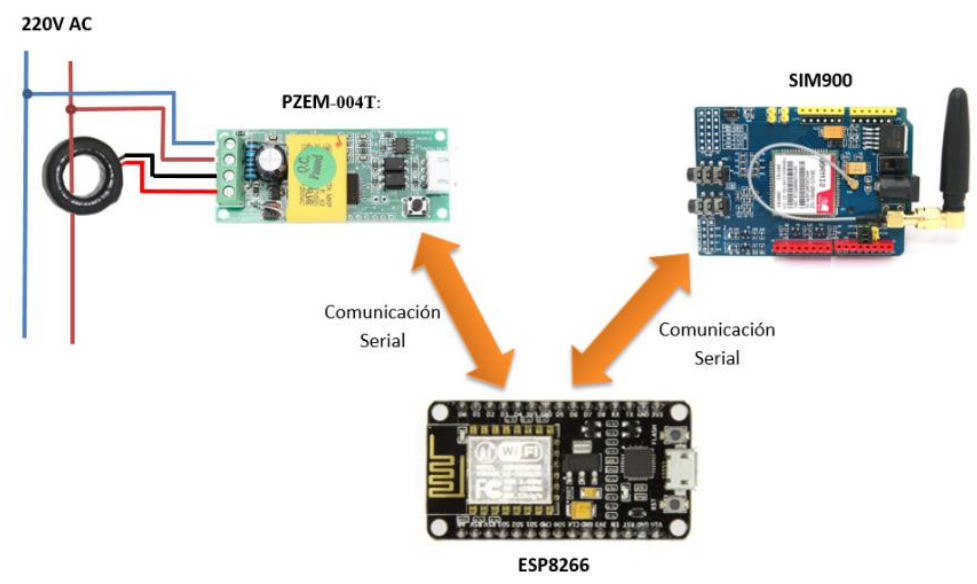

Figura VI - Estructura Operativa del sistema de medición 
El proceso de obtención de datos se lleva a cabo de la siguiente manera:

En primer lugar, el sensor de corriente y voltaje censa la línea de entrada de energía eléctrica del domicilio, este dispositivo utilizando un circuito electrónico dedicado, procesa la información y la envía mediante una comunicación serial al módulo del microcontrolador ESP8266, este es capaz de interpretar los datos y generar la trama que será enviada al servidor en Internet.

Dicha trama es enviada por otro puerto de comunicaciones seriales al módulo transceptor SIM900, siendo este el encargado de conectarse vía inalámbrica con la red GPRS correspondiente al operador de la red celular acorde al chip GSM que se haya instalado, este último lleva la información a internet y por consiguiente al servidor en cuestión donde se almacenaran y procesaran los datos, obteniendo como resultado la información necesaria para poder decidir qué acciones son las más adecuadas para recomendarle al usuario en pro de reducir el consumo eléctrico.

3.2. Costo del dispositivo.- En la tabla I se detalla el costo en dólares americanos de cada uno de los componentes utilizados para crear el prototipo.

\begin{tabular}{|l|r|}
\hline Ítem & Costo \\
\hline PZEM-004T & USD 5.00 \\
\hline sim900 & USD 17.00 \\
\hline ESP8266 & USD 5.00 \\
\hline gabinete & USD 3.00 \\
\hline cables y misceláneas & USD 2.00 \\
\hline Total & USD 32.00 \\
\hline
\end{tabular}

Tabla I.-Costos de los componentes del dispositivo.

3.3. Pruebas técnicas.- El primer objetivo fue verificar el correcto funcionamiento de todo el sistema en conjunto, incluyendo el dispositivo de medición, la red de comunicaciones y el servidor junto con la base de datos alojada en la nube donde se almacena, procesa y accede a la información de telemetría.

Luego de esto se procedió a validar si la información recolectada en las pruebas podría ser de utilidad para comprender patrones de consumo eléctrico de parte del usuario que puedan asociarse a oportunidades de ahorro. Esto se realizó a través del análisis de los datos de consumo recogidos y su relación con algunos de los casos de uso expuestos en la primera parte del trabajo.

En este siguiente apartado se explican en detalle las pruebas técnicas realizadas para corroborar el correcto funcionamiento del dispositivo desarrollado y se muestran los resultados de los análisis de datos realizados sobre la información recolectada.

3.3.2. Preparación de las pruebas.- Se planificó la realización de pruebas en dos domicilios diferentes de la Ciudad de Córdoba, Argentina durante un mes completo. Ambos domicilios contaban con servicio eléctrico monofásico de $220 \mathrm{~V}$.

\section{$\underline{\text { Conexión del dispositivo }}$}

El dispositivo se conectó con su bobina toroide al cable de vivo del hogar, con el fin de medir la potencia consumida. El diagrama de conexión se ilustra en la figura VII: 


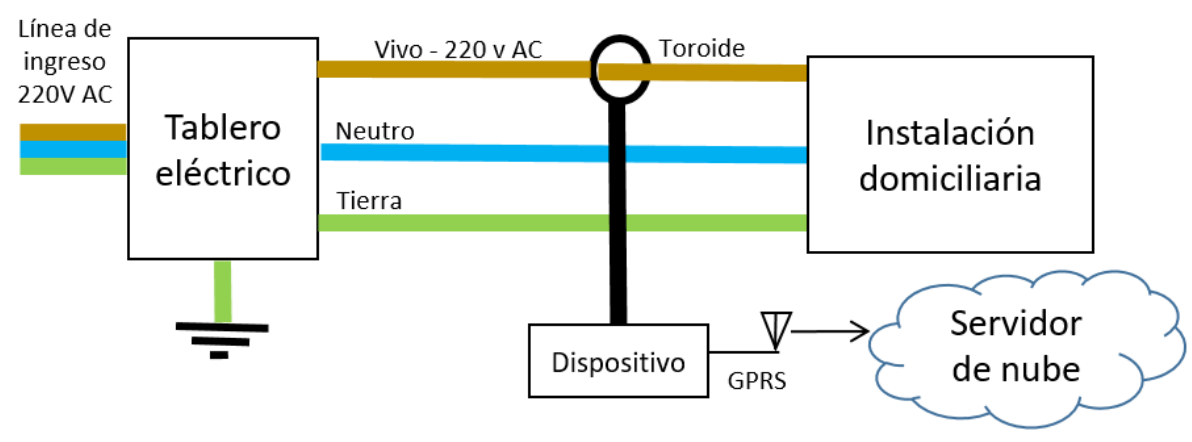

Figura VII.-Esquema de conexión de dispositivo de lectura.

Las mediciones efectuadas por el dispositivo se enviaron a través de un canal de conexión GPRS de un proveedor de telefonía celular, mediante el uso del protocolo MQTT, al servidor de nube para que almacene los datos en la base de datos.

Al finalizar el período de prueba se realizó una exportación de datos a formato de texto .csv y se utilizó la herramienta Tableau en su versión pública [12] para realizar los análisis pertinentes. El archivo de datos contiene un registro por cada medición, con el siguiente formato:

- El primer campo indica la fecha, Formato dd/mm/aaaa.

- El segundo campo contiene el horario de la medición en formato hh:mm de 24 hs.

- El tercer campo muestra la potencia real, calculada a través de la medición de corriente instantánea medida en el dispositivo.

3.3.3. Perfil de consumo de los domicilios.- La tabla II muestra los dispositivos existentes en cada uno de los domicilios.

\begin{tabular}{|l|l|}
\hline DOMICILIO 1 & DOMICILIO 2 \\
\hline Heladera & Heladera \\
\hline Televisores Led (3) & Freezer \\
\hline Horno Microondas & Televisores Led (3) \\
\hline Pava eléctrica & Horno Microondas \\
\hline Lavarropas & Horno Eléctrico \\
\hline Computadora Personal & Pava eléctrica \\
\hline Notebooks (2) & Lavarropas \\
\hline Aire Acondicionado Frio/Calor (2) & Freidora \\
\hline Calefactor eléctrico (1 & Computadora Personal \\
\hline Luminarias Bajo consumo (15) & Notebooks (3) \\
\hline Central de alarma & Aire Acondicionado Frio/Calor (1) \\
\hline Router Wifi (1) & Calefactor eléctrico (2) \\
\hline Celulares/Tablets con cargadores (3) & Luminarias Bajo consumo (20) \\
\hline & Central de alarma \\
\hline & Router Wifi (1) \\
\hline
\end{tabular}

Tabla II.- Dispositivos eléctricos por domicilio. 


\subsubsection{Desarrollo de las pruebas.-}

\section{Pruebas en Domicilio 1}

El período de recolección de datos: Desde el 01/05/2018 18:10 al 31/5/2018 23:59 en intervalos de 1 minuto por medición. Trascurrido este período se analizaron las mediciones y se obtuvieron los siguientes resultados ilustrados en la figura VIII:

En primera instancia se obtiene la curva de consumo de ambos períodos en promedios diarios de consumo, visualizando en dos colores diferentes según sea Día hábil o Feriado.

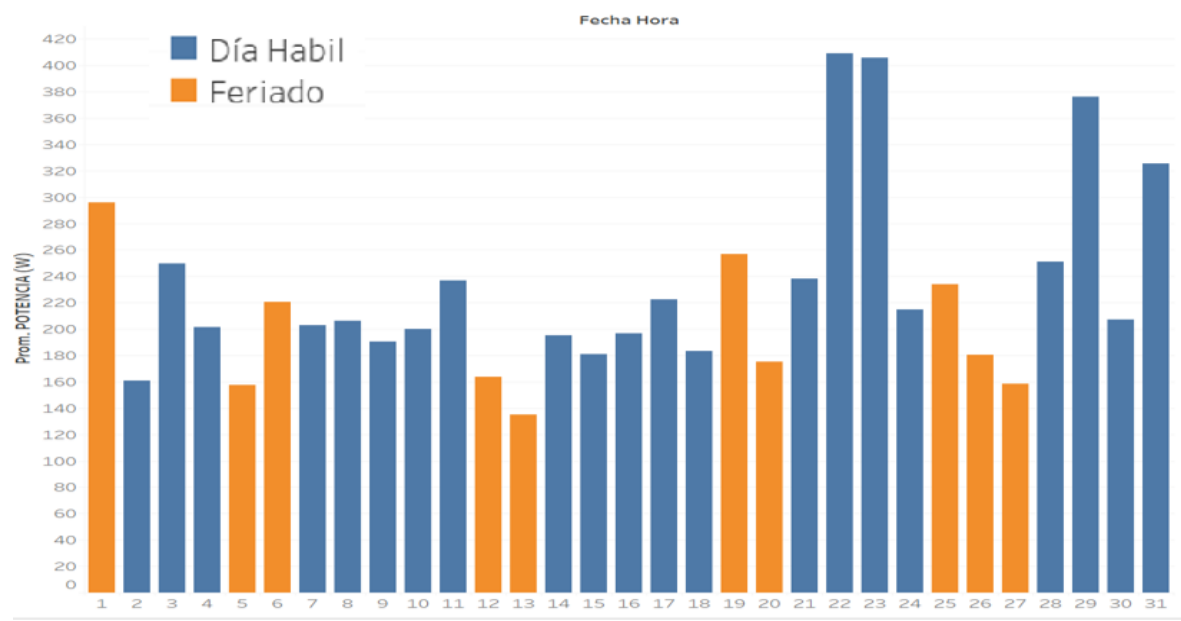

Figura VIII.- Energía total consumida en el día - Domicilio 1.

A priori se observa que el consumo de los días hábiles en general es mayor que en los feriados y que el consumo tiende a subir hacia fin de mes. Por tratarse de un mes de otoño es posible que esto esté mostrando mayor uso de sistemas de calefacción eléctrico. Se verifica que el día de menor consumo fue el 13/5 (sábado) y el de mayor consumo el 22/5 (lunes).

Se procede luego a analizar la curva de consumo según la hora del día, tanto para días hábiles como para días feriados (Figura IX) donde se visualizan 3 momentos de picos, coincidentes con las 6:00, las 11:00 y las 20:00 hs, siendo el de las 11:00 el más alto con $300 \mathrm{~W}$. Por otra parte, el consumo menor se presenta a las 5:00, 9:00 y 17:00 hs. con menos de $150 \mathrm{~W}$ promedio. Cuando se analiza el gráfico disgregado por día hábil y feriados se observan las siguientes características:

- Pico máximo día hábil : 20 hs $-450 \mathrm{~W}$

- Pico máximo feriado : 18 hs - $250 \mathrm{~W}$

- Primer pico día hábil : 06 hs - $270 \mathrm{~W}$ (despertar)

- Primer pico día feriado : 09 hs. $220 \mathrm{~W}$ (despertar)

- Consumo mínimo día hábil : 05 hs. $-150 \mathrm{~W}$

- Consumo mínimo feriado : 08 hs. $-130 \mathrm{~W}$ 


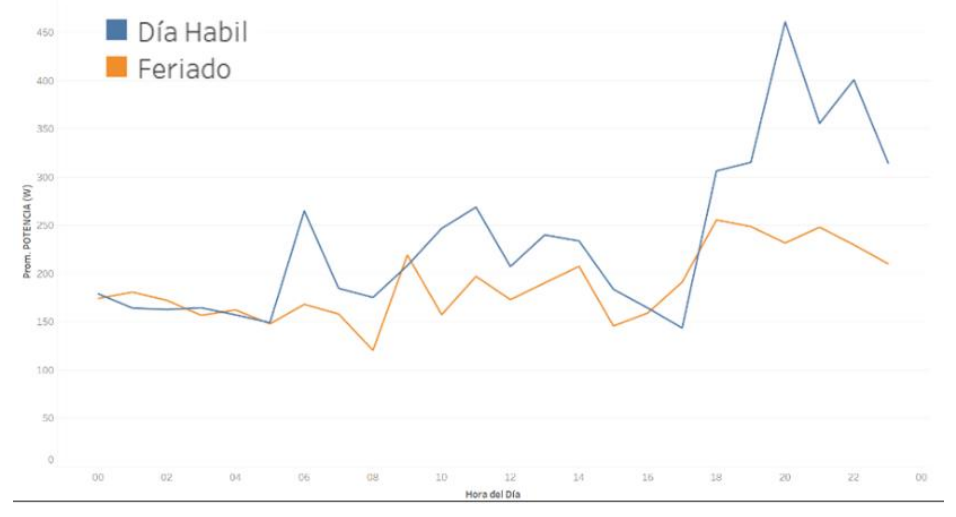

Figura IX.-Consumo promedio por hora del día-Domicilio 1.

Por último, se realizaron análisis de consumo en 3 días determinados (mayor consumo, menor consumo y cercano al promedio) en busca de patrones que puedan indicarnos perfiles o conductas de consumo.

En el primer día analizado (mayor consumo) ilustrado en la figura X se pueden detectar algunos datos importantes, a saber.

El consumo mínimo de este hogar rondaría los $100 \mathrm{~W}$, que formaría parte de los dispositivos "siempre encendidos" como por ejemplo luz de led testigo de dispositivos electrónicos, routers de Internet, sensores de alarmas, etc. (flecha roja)

Se puede visualizar la curva de consumo de la heladera, en especial durante las horas de la madrugada, ya que es el único elemento inductivo que permanece encendido y se activa por períodos casi constantes en el tiempo (flecha verde).

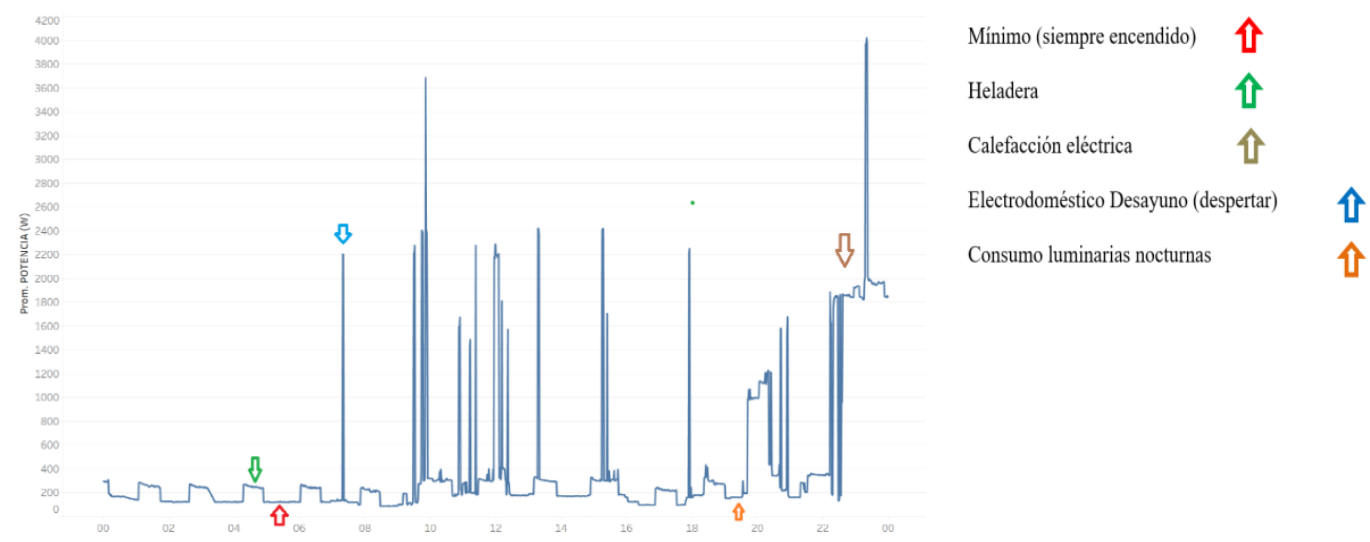

Figura X.- Día de mayor consumo - martes 22/5 - Domicilio 1.

Adicionalmente se puede inferir el consumo de los elementos lumínicos del hogar, dados por la diferencia en los valles de la curva de consumo a partir de las 19:30 hs., y los producidos en horarios diurnos (flecha naranja). 
Los picos en forma de aguja parecen ser elementos de alto consumo, quizás asociados a calefacción eléctrica o electrodomésticos hogareños. El primer pico del día se infiere como electrodoméstico de desayuno (flecha azul).

Los altos consumos nocturnos hacen referencia a la calefacción eléctrica, más teniendo en cuenta la época del año (flecha marrón).

Se analizan luego otros días subsiguientes para corroborar si se repiten las observaciones.

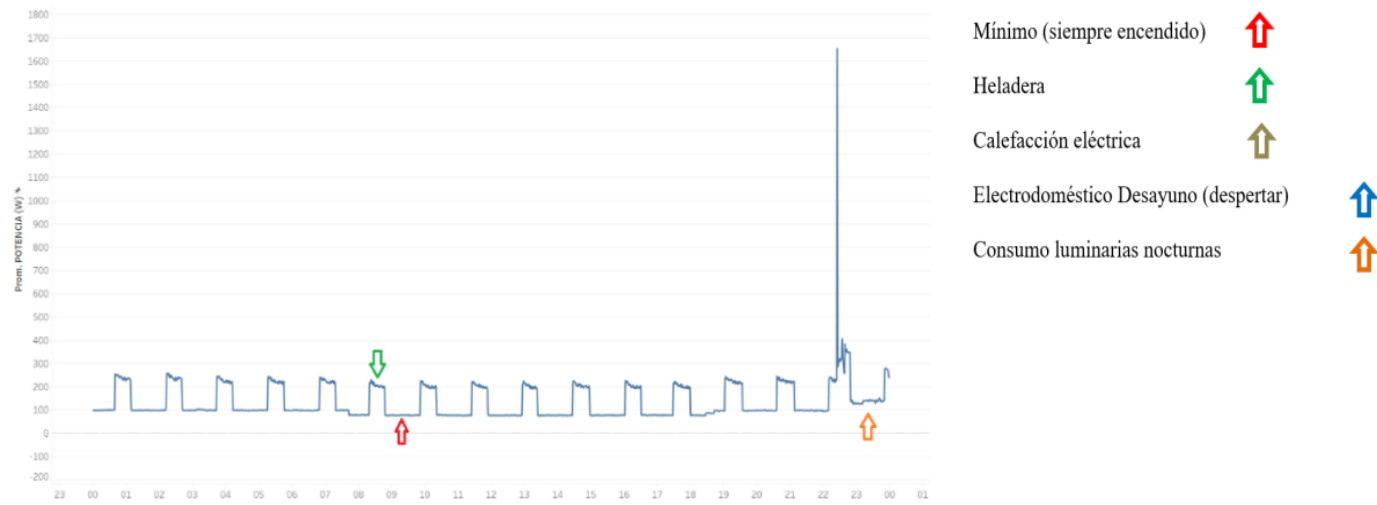

Figura XI.-Día de menor consumo - domingo 13/5 - Domicilio 1.

Al observar la curva del día de menor consumo (13/5) en la figura XI se observa que el primer consumo en forma de aguja recién se verifica pasadas las 22:00 lo que podría indicar ausencia del hogar de las personas durante el día. Se corrobora patrón de consumo de la heladera, el consumo mínimo y el patrón de luces encendidas.

Por último, se analizó un día que presentara un valor de consumo cercano al promedio. En este caso se eligió el 10/05 (jueves) ilustrado en la figura XII.

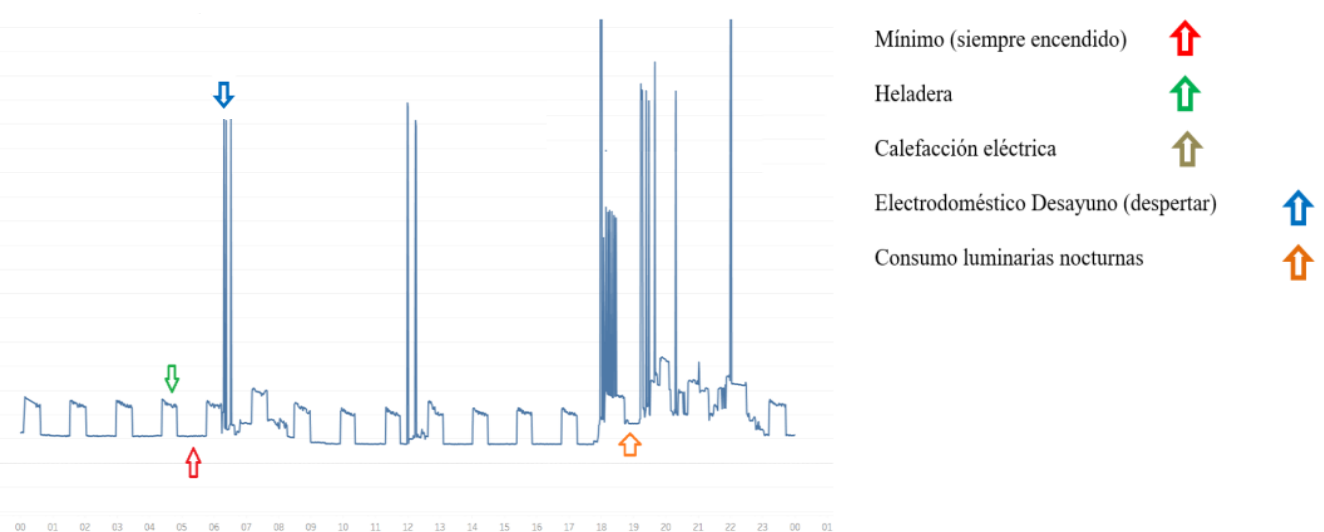

Figura XII.- Consumo del día jueves 10/5 - Domicilio 1. 
Se corrobora patrón de consumo de la heladera como así también consumo mínimo. Se observan patrones de consumo en forma de aguja por la mañana (desayuno) y consumo lumínico nocturno (flecha naranja) como piso elevado a partir del anochecer (19 hs.)

A partir de las observaciones obtenidas se podría comenzar a inferir el consumo de los dispositivos hogareños, por ej. en este caso se observa que los dispositivos que siempre permanecen encendidos en este hogar tienen un consumo aproximado de $50 \mathrm{~W}$ y la heladera unos $100 \mathrm{~W}$ cada vez que enciende el motor.

\section{$\underline{\text { Confiabilidad del sistema }}$}

Se procedió a analizar la cantidad de minutos en el período sobre el cual no se pudieron contar con mediciones. En la tabla III se muestra el grado de asertividad de las mediciones.

\begin{tabular}{|c|r|}
\hline Total & 42110 \\
\hline Efectivas & 35206 \\
\hline Errores & 6904 \\
\hline Porcentaje de eficiencia & $83,60 \%$ \\
\hline
\end{tabular}

Tabla III.-Mediciones efectivas - Domicilio 1.

Se pudo observar momentos en los cuales no se registran mediciones, siendo el porcentaje de mediciones obtenidas sobre el total de mediciones posibles de $83,60 \%$.

Si bien este nivel de asertividad es más que suficiente para el objetivo de un medidor hogareño de bajo costo, donde su principal función es el de dar a conocer a usuario información de consumo que sirva para optimizar el uso de la energía eléctrica, de cara a la segunda prueba se evaluaron acciones tendientes a mejorar esta tasa de efectividad.

En primer lugar, se buscó incrementar la confiabilidad del dispositivo a fin de aumentar los valores de eficiencia, para de este modo lograr un correcto análisis de consumo. Para ello se realizaron mejoras en el firmware tendientes a incrementar la seguridad en la comunicación de los datos.

Por otra parte, se evaluó la posibilidad de aumentar el tiempo de recolección de datos. Realizando el análisis de las mediciones recolectadas se verifica que es posible realizar una correcta interpretación de los mismos aun cuando el intervalo de medición pase de 1 minuto, como el implementado en las pruebas en el domicilio 1, a 5 minutos.

A pesar de que hay una pérdida de datos en las mediciones estas aún son suficientes para el análisis y redunda en importantes beneficios para el sistema, en primera instancia al utilizarse comunicaciones móviles para el envío de datos, las mismas están tarifadas en relación a la cantidad de información transferida, con este nuevo intervalo de medición esta se reduce en cinco veces.

Otro importante beneficio derivado del aumento en el intervalo de medición es que si se trata de un sistema de medición a gran escala con gran cantidad de sensores se estaría en presencia de un importante volumen de datos con los consabidos costos para almacenar los mismos en los servidores, es por ello que una reducción en la cantidad de datos también llevaría asociada una 
reducción en los costos de almacenamiento, y si ello no afecta el rendimiento del sistema es evidente que se deben incluir estas mejoras.

\section{Pruebas en Domicilio 2}

Período de recolección de datos: Desde el 01/08/2018 00:00 a 31/08/2018 23:59 en intervalo de 5 minutos entre mediciones.

Para comenzar se analizó la curva de promedios diarios de consumo en el mes, visualizando en dos colores diferentes según sea Día hábil o Feriado. Ilustrado en la figura XIII.

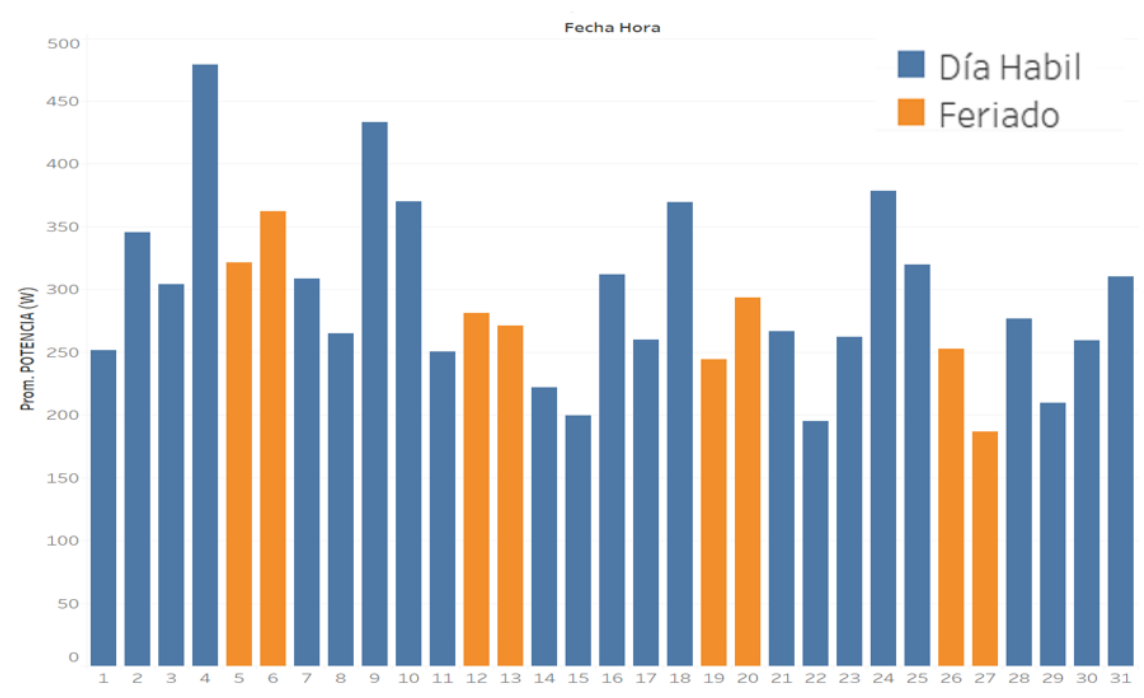

Figura XIII.- Energía total consumida en el día-Domicilio 2.

Al igual que en el primer domicilio el valor observado en los días hábiles en general es mayor que en los feriados, pero en este caso se ve que consumo tiende a bajar hacia fin de mes. Por tratarse del mes de agosto es posible que esto esté mostrando un menor uso de sistemas de calefacción eléctricos en los últimos días del mes. Se verifica además que el día de menor consumo es el 27/8 (domingo) y el de mayor consumo el 4/8 (viernes).

Luego de esto se continuó con el estudio de la curva de consumo promedio según la hora del día, tanto para días hábiles como para días feriados ilustrado en la figura XIV. Para el caso de los días hábiles se visualizan 3 momentos de picos, coincidentes con las 8:00 hs., las 13:00 hs. y las 21:00 hs., siendo este último el más alto con $550 \mathrm{~W}$.

Como contraparte, el menor consumo se presenta a las 06:00 hs. con menos de $150 \mathrm{~W}$ promedio y para el caso de los días feriados sólo se presenta un pico marcado en horario nocturno. 


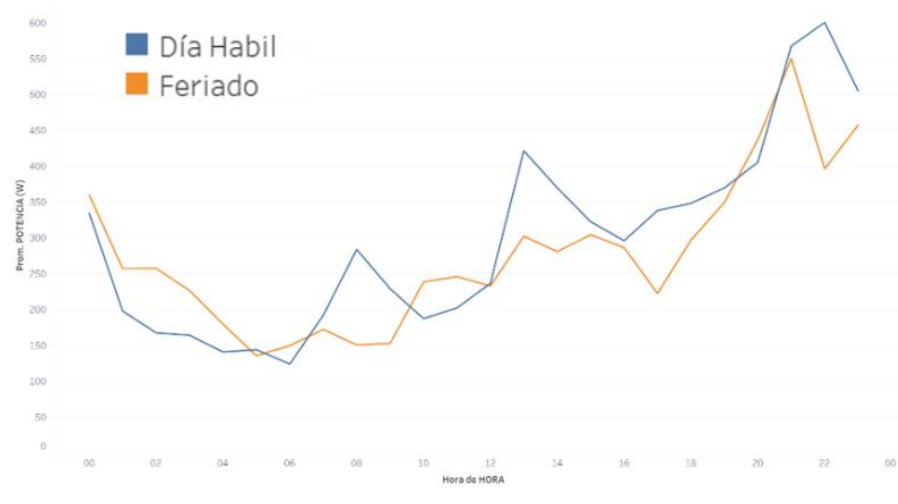

Figura XIV.-Consumo promedio por hora del día-Domicilio 2.

Para finalizar se revisaron las curvas correspondientes a 3 días determinados (el de mayor consumo ilustrado en la figura XV, el de menor ilustrado en la figura XVI y el de un día cercano al promedio ilustrado en la figura XVII) en busca de patrones que puedan indicarnos perfiles o conductas de consumo. Los resultados son los siguientes:

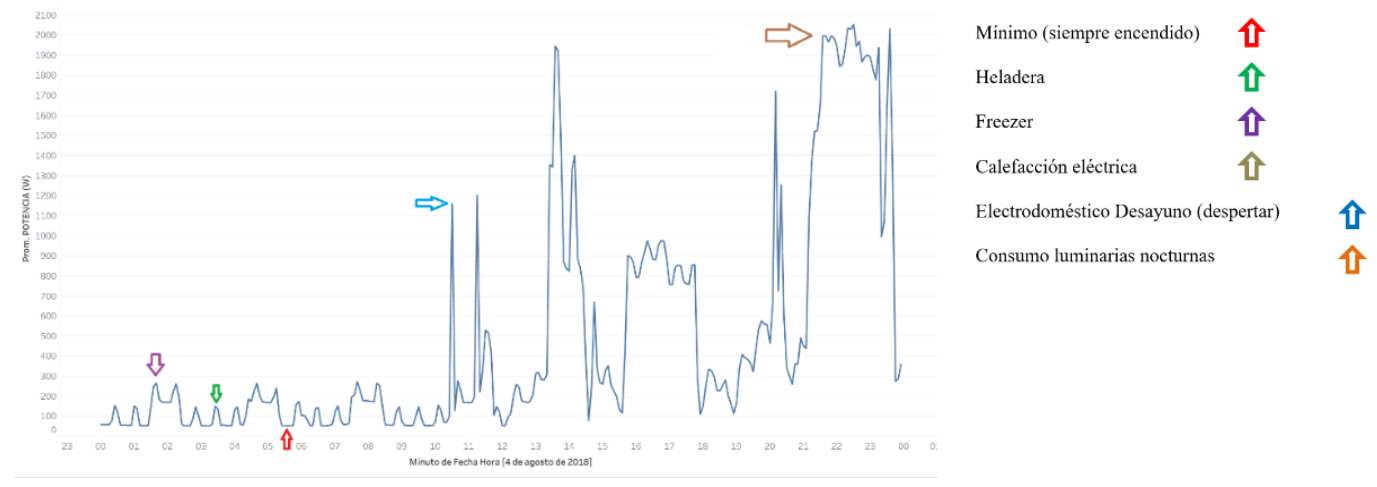

Figura XV.-Día de mayor consumo - viernes 4/8 - Domicilio 2.

En el primer día analizado se pueden detectar algunos datos importantes, a saber.

1) El consumo mínimo de este hogar está alrededor de $100 \mathrm{~W}$, conformado por los dispositivos "siempre encendidos" como por ejemplo luz de led testigo de dispositivos, routers de Internet, sensores de alarmas, etc (flecha roja).

2) Se puede inferir claramente el perfil de consumo de las heladeras y el freezer, en especial durante las horas de la madrugada, ya que son los únicos elementos inductivos encendidos y se activan por períodos casi constantes en el tiempo (flecha verde y morada).

3) Los picos en forma de aguja parecen ser elementos de alto consumo, quizás asociados a calefacción eléctrica o electrodomésticos hogareños. El primer pico del día se infiere como electrodoméstico de desayuno, como pava eléctrica o cafetera (flecha azul).

4) Los altos consumos nocturnos hacen referencia a la calefacción eléctrica, más teniendo en cuenta la época del año (flecha marrón). 
Se continuó con el análisis del resto de los días para corroborar si se repiten las observaciones.

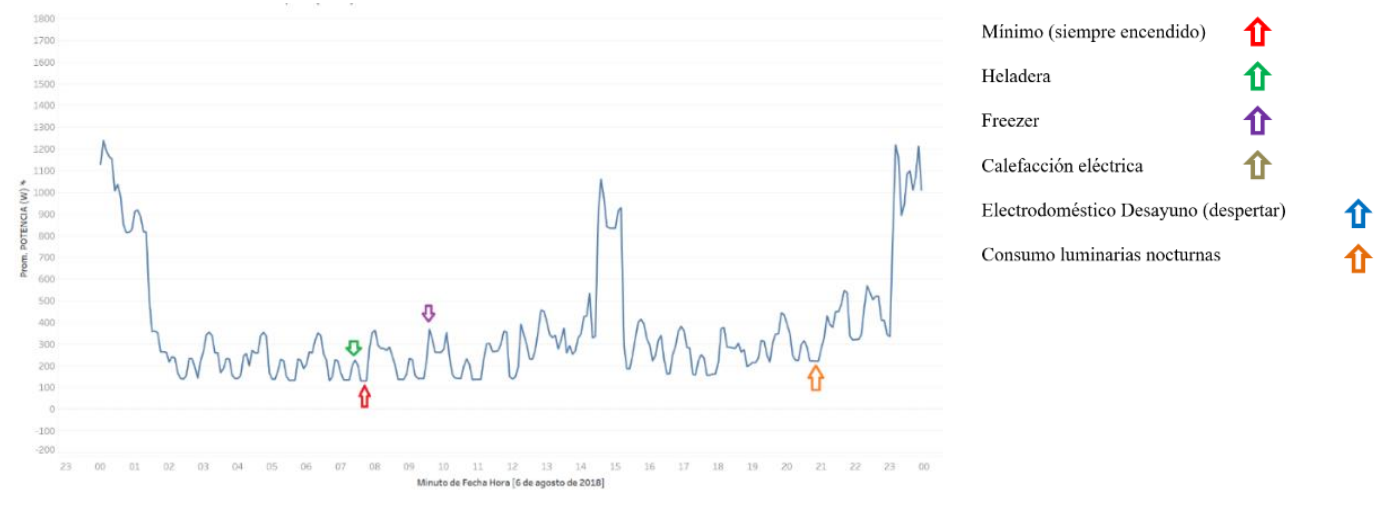

Figura XVI.- Día de menor consumo - domingo 27/8 - Domicilio 2.

En este caso se verificó nuevamente el patrón de consumo de la heladera y freezer, como así también el consumo mínimo. En cambio, no se visualizan altos patrones de consumo nocturnos (calefacción eléctrica) ni consumo en forma de aguja por la mañana (desayuno), lo que podría indicar desocupación de la vivienda hasta el mediodía. Por otra parte, se presenta el patrón de consumo lumínico nocturno (flecha naranja) como piso elevado a partir del anochecer.

Por último, se analiza la curva de consumo de un día cercano al promedio. En este caso elegimos el 16/08 (jueves).

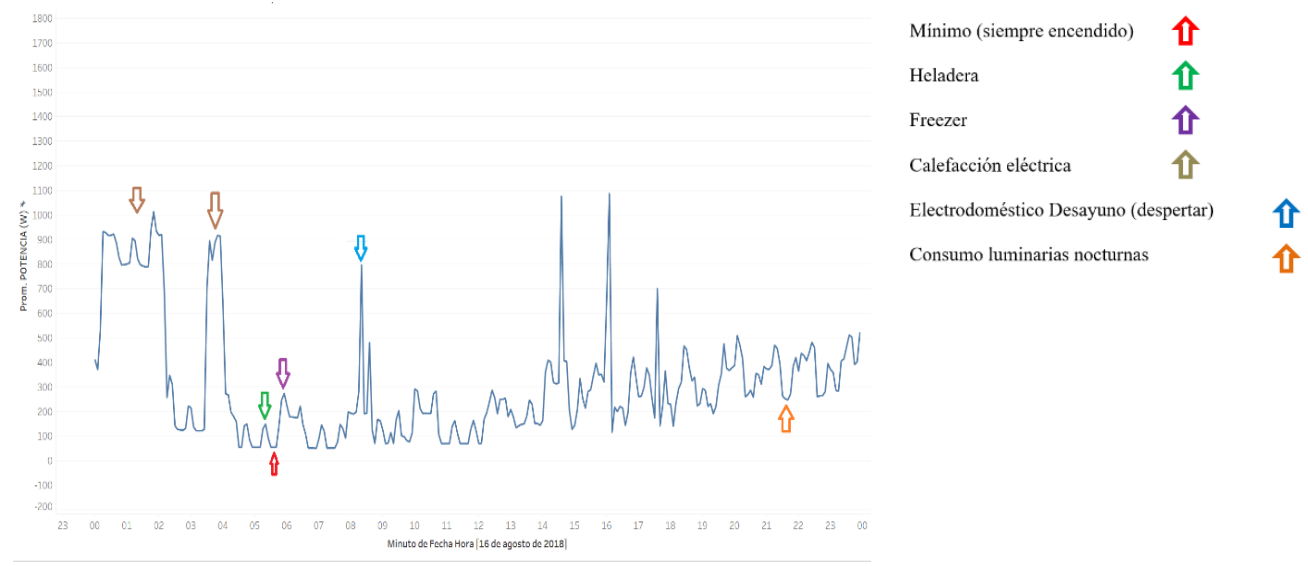

Figura XVII.- Consumo del día jueves 16/08-Domicilio 2.

Se presenta el patrón de consumo de la heladera y el freezer como en los casos anteriores, como así también el nivel de consumo mínimo. Se observa además consumos nocturnos (calefacción eléctrica) y en forma de aguja por la mañana (desayuno). Por último, se verifica el patrón de consumo lumínico nocturno (flecha naranja) como piso elevado a partir del anochecer $(20 \mathrm{hs}$.). 
Como el objetivo de las pruebas no es el de realizar cálculos de consumo, sino el de demostrar que las mediciones obtenidas por el dispositivo pueden ser utilizadas para dicho fin, no se continuó con el análisis más allá de lo expuesto.

\section{$\underline{\text { Confiabilidad del Sistema }}$}

Al igual que en el primer caso se procedió a analizar la cantidad de minutos en el período sobre el cual no se pudieron contar con mediciones. En la tabla IV se muestra el grado de asertividad de las mediciones.

\begin{tabular}{|l|r|}
\hline Total & 8928 \\
\hline Efectivas & 8922 \\
\hline Errores & 6 \\
\hline Porcentaje de eficiencia & $99,93 \%$ \\
\hline
\end{tabular}

Tabla IV.-Mediciones efectivas - Domicilio 2.

Se puede observar una efectividad a un $99,93 \%$, en parte por la extensión del período de muestreo de 1 a 5 minuto. Si bien al tener un mayor período entre mediciones se pierde algo de precisión, se pudo verificar que los patrones observados en el caso 1 se pueden detectar sin problemas en el segundo caso, por lo que se considera que el periodo de 5 minutos es totalmente aplicable para este tipo de sensores y quizás más apropiado por permitir una reducción importante de costo de trasmisión y almacenamiento.

4. Conclusiones y resultados.- Como resultado del trabajo se ha logrado definir un prototipo técnico de recolección y análisis de datos de consumo de energía eléctrica soportado por una arquitectura tecnológica de nube que hizo posible la colección de información en un sistema que pueda manejar grandes cantidades de datos para su posterior análisis y visualización. Todo esto ha sido realizado con tecnologías abiertas disponibles en el mercado a un costo considerablemente bajo, lo que permite ser replicado fácilmente y adaptado para su uso en hogares, edificios u organizaciones con poco esfuerzo.

Por otra parte se pudieron describir diversos casos de uso donde el análisis de los datos recolectados puedan ser aplicados en distintas acciones destinadas a conseguir un uso de la energía más eficiente, ya sea para el descubrimiento y análisis de patrones de consumo que permitan detectar anomalías o conductas inapropiadas como así también para la determinación de predicciones que posibiliten anticiparse a las demandas.

Por todo lo expuesto es deseable que este trabajo pueda ser de ayuda al abordaje de la problemática del uso de tecnología de big data para lograr eficiencia energética a través de su aplicación práctica con sistemas abiertos, y además pueda colaborar aportando entendimiento sobre algunos de las múltiples aplicaciones donde esta metodología puede aplicarse en pos de mejorar las conductas de consumo de la sociedad. 


\section{Bibliografía}

[1] Comisión Económica para Latinoamérica y el Caribe (CEPAL). "Indicadores de políticas públicas en materia de eficiencia energética en América latina y el Caribe”, 2010

[2] Martinez A. P. "Modelo de predicción de la demanda eléctrica mediante regresión dinámica". Universidad Polítécnica de Madrid, España, 2015

[3] González-Avella J.C y Tuduri J.M., "Análisis de series temporales usando redes neuronales". Bareales, España, 2017.

[4] Gabriel Natán Pizarro Herrera, "Reconocimiento de patrones y pronóstico de consumo eléctrico". Universidad Católica De Valparaíso, Chile 2017.

[5] Santiago de la Fuente Fernandez, "Series Temporales: Modelo ARIMA". Universidad Autónoma de Madrid. España, 2013.

[6] Guillermo Beyrne, Facundo Malvicino, Luis Alberto Trajtenberg. "Modelo Estacional de Demanda de Energía Eléctrica". Secretaría de Política Económica y Planificación del Desarrollo, Argentina 2015.

[7] Instituto Tecnológico de Massachusetts (MIT), Base de datos Open Source para series temporales. https://docs.influxdata.com/influxdb , 2018.

[8] Instituto Tecnológico de Massachusetts (MIT), Framework para desarrollo de aplicaciones Web PHP. https://laravel.com/docs , 2018.

[9] MariaDB Foundation, Proyecto de Base de Datos relacional. https://mariadb.org/documentation/, 2018.

[10] Oracle Corp., Proyecto de Base de Datos relacional Open Source. https://www.mysql.com/docs, 2018.

[11] Arduino AG., Plataforma de creación de electrónica de código abierto. https://www.arduino.cc/,, 2018.

[12] Tableau Software LLC., Plataforma gratuita en línea para explorar visualizaciones de datos. https://public.tableau.com/, 2018. 